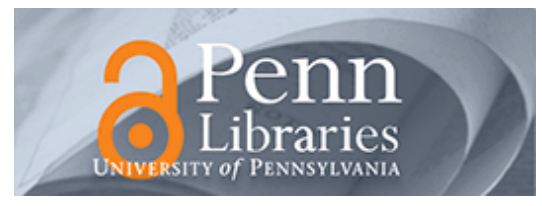

University of Pennsylvania

ScholarlyCommons

March 2003

\title{
Hyperspectral Image Processing Using Locally Linear Embedding
}

\author{
David H. Kim \\ University of Pennsylvania \\ Leif H. Finkel \\ University of Pennsylvania, leif@neuroengineering.upenn.edu
}

Follow this and additional works at: https://repository.upenn.edu/be_papers

\section{Recommended Citation}

Kim, D. H., \& Finkel, L. H. (2003). Hyperspectral Image Processing Using Locally Linear Embedding. Retrieved from https://repository.upenn.edu/be_papers/8

Copyright 2003 IEEE. Reprinted from Proceedings of the 1st International IEEE EMBS Conference on Neural Engineering 2003, pages 316-319.

Publisher URL: http://ieeexplore.ieee.org/xpl/tocresult.jsp?isNumber=26900\&page=5

This material is posted here with permission of the IEEE. Such permission of the IEEE does not in any way imply IEEE endorsement of any of the University of Pennsylvania's products or services. Internal or personal use of this material is permitted. However, permission to reprint/republish this material for advertising or promotional purposes or for creating new collective works for resale or redistribution must be obtained from the IEEE by writing to pubs-permissions@ieee.org. By choosing to view this document, you agree to all provisions of the copyright laws protecting it.

This paper is posted at ScholarlyCommons. https://repository.upenn.edu/be_papers/8

For more information, please contact repository@pobox.upenn.edu. 


\title{
Hyperspectral Image Processing Using Locally Linear Embedding
}

\begin{abstract}
We describe a method of processing hyperspectral images of natural scenes that uses a combination of k-means clustering and locally linear embedding (LLE). The primary goal is to assist anomaly detection by preserving spectral uniqueness among the pixels. In order to reduce redundancy among the pixels, adjacent pixels which are spectrally similar are grouped using the k-means clustering algorithm. Representative pixels from each cluster are chosen and passed to the LLE algorithm, where the high dimensional spectral vectors are encoded by a low dimensional mapping. Finally, monochromatic and trichromatic images are constructed from the k-means cluster assignments and LLE vector mappings. The method generates images where differences in the original spectra are reflected in differences in the output vector assignments. An additional benefit of mapping to a lower dimensional space is reduced data size. When spectral irregularities are added to a patch of the hyperspectral images, again the method successfully generated color assignments that detected the changes in the spectra.

Keywords

hyperspectral image, locally linear embedding, anomaly detection

\section{Comments}

Copyright 2003 IEEE. Reprinted from Proceedings of the 1st International IEEE EMBS Conference on Neural Engineering 2003, pages 316-319.

Publisher URL: http://ieeexplore.ieee.org/xpl/tocresult.jsp?isNumber=26900\&page=5

This material is posted here with permission of the IEEE. Such permission of the IEEE does not in any way imply IEEE endorsement of any of the University of Pennsylvania's products or services. Internal or personal use of this material is permitted. However, permission to reprint/republish this material for advertising or promotional purposes or for creating new collective works for resale or redistribution must be obtained from the IEEE by writing to pubs-permissions@ieee.org. By choosing to view this document, you agree to all provisions of the copyright laws protecting it.
\end{abstract}




\title{
Hyperspectral Image Processing Using Locally Linear Embedding
}

\author{
David H. Kim, Leif H. Finkel
}

Department of Bioengineering, University of Pennsylvania, Philadelphia, PA, USA

\begin{abstract}
We describe a method of processing hyperspectral images of natural scenes that uses a combination of $k$-means clustering and locally linear embedding (LLE). The primary goal is to assist anomaly detection by preserving spectral uniqueness among the pixels. In order to reduce redundancy among the pixels, adjacent pixels which are spectrally similar are grouped using the k-means clustering algorithm. Representative pixels from each cluster are chosen and passed to the LLE algorithm, where the high dimensional spectral vectors are encoded by a low dimensional mapping. Finally, monochromatic and tri-chromatic images are constructed from the $k$-means cluster assignments and LLE vector mappings. The method generates images where differences in the original spectra are reflected in differences in the output vector assignments. An additional benefit of mapping to a lower dimensional space is reduced data size. When spectral irregularities are added to a patch of the hyperspectral images, again the method successfully generated color assignments that detected the changes in the spectra.
\end{abstract}

Keywords - hyperspectral image, locally linear embedding, anomaly detection

\section{INTRODUCTION}

Unlike RGB images which are only tri-chromatic, hyperspectral images are composed of large numbers of color planes, each corresponding to a different wavelength in the electromagnetic spectrum. Hyperspectral images typically contain anywhere from tens to hundreds of spectral bands and can range any segment of the electromagnetic spectrum, but usually span at least the visible spectrum from infrared to ultraviolet. Increasing the number of spectral channels results in a finer spectral resolution, which is usefu in discriminating between various materials in a scene, since each substance has unique radiance and reflectance spectra. Satellite hyperspectral imagery finds many applications where accurate identification of compounds and features on the earth's surface is important. Identification of useful minerals can lead to efficient mining [4], and understanding spectra of farm crops at various stages of growth can lead to more efficient water and fertilizer usage [6-7].

Although hyperspectral imagery provides a wealth of information about a scene, a drawback of the increased spectral resolution is the large size of the dataset generated for each image. The large datasets are cumbersome, impede data transmission and make data mining difficult. To complicate matters, as imaging technology improves, spatial resolution, spectral resolution and bit-depth will only

This work was supported by the DoD Multidisciplinary University Research Initiative (MURI) program administered by the Office of Naval Research under Grant N00014-01-1-0625. increase, resulting in ever larger image sizes.

The method proposed here attempts to address the issues of anomaly detection and data compression by applying the locally linear embedding (LLE) algorithm [1] to hyperspectral images. Unlike other dimensional scaling techniques such as multidimensional scaling (MDS) and Isomap [5] which depend on measuring pair-wise distances among data points to compute embeddings, LLE computes embeddings from locally linear fits. LLE is a nonlinear algorithm used to reduce the dimensionality of high dimensional data while preserving local geometries in a lowdimensional representation of the original data. Given a well sampled high-dimensional manifold, the algorithm will map the data points relative to the manifold and local relationships remain. In the case of hyperspectral anomaly detection, LLE maps spectra that are similar to low dimensional outputs that are similar and maps spectra that are dissimilar to low-dimensional outputs that are dissimilar. Thus, in principle, materials with unique spectral signatures could be distinguished in the image.

The LLE algorithm consists of three steps [1]. First, a predetermined number of neighbors are assigned to each data point. Neighbors can be defined by any method, such as nearest neighbors by Euclidian distance. Second, weights are computed for each point that best characterize local geometries. Third, the embeddings are computed from the weights to minimize reconstruction error. The result is the high dimensional data encoded into a low dimensional space.

\section{METHODOLOGY}

The hyperspectral images used in this study are described in [2] and were captured with a hyperspectral camera. The camera by itself captured monochromatic images, but narrow-band filters used with the camera filtered the incoming light before hitting the camera's CCD. The filters were changed for each color channel. The pictures are of natural scenes such as landscapes along the English countryside as well as close-up pictures of leaves and wooded areas. In every scene is a white flag that was used for image calibration. Each hyperspectral image consists of a series of 31 chromatically narrow-band-filtered 8-bit $256 \times 256$ pixel images ranging spectrally from $400 \mathrm{~nm}$ to $700 \mathrm{~nm}$.

The k-means algorithm was used to pre-process the images. The image was segmented into clusters and a representative pixel was chosen from the center of each cluster. Using k-means to segment the image had two advantages. First, we were able to indirectly add a threshold for pixel similarity. By varying the number of clusters, the 


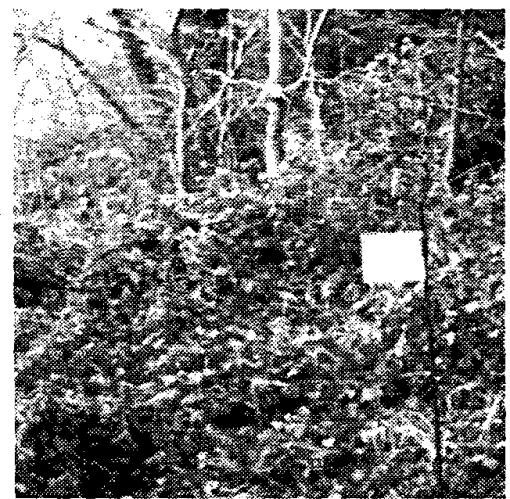

Fig.1 - A sample hyperspectral image converted to RGB.

algorithm controls the similarity among clustered pixels. Increasing the number of bins makes the clustering more discriminating and decreasing the number of bins increases the tolerance for dissimilar pixels. The second advantage was that by grouping several similar pixels, we reduced the number of vectors sent to the next stage. We found that the LLE algorithm required more computer resources than the clustering algorithm, so reducing the load on the LLE algorithm was advantageous. By reducing the size of the vector list, we improved the efficiency of the overall algorithm and decreased the demand on computer resources. These in turn allowed us to process larger images and improve the output image resolution.

Next, the LLE algorithm was used to reduce the dimensionality of the spectra. The dimensionality of the output was a free parameter. We chose to reduce the spectra to both one dimension as well as three dimensions. The one dimensional output was simplest and would show obvious image features in grayscale. The three-dimensional output spectra provided a larger color-space and it could also be easily displayed using by arbitrarily assigning each of the output channels to RGB.

Finally, the image was reconstructed from the k-means cluster map and the LLE pixel vector mapping. We obtained final images by combining $\mathrm{k}$-means cluster-to-pixel mappings and the spectra-to-short vector mappings. The end result was an image where the spectrum from each pixel in the hyperspectral image was mapped to a unique lowdimensional vector in the output image and the variation in spectra was reflected the differences in the output color.

Although the original images were images that were $256 \times 256$ pixels, the maximum output resolution achieved on an Athlon $1200 \mathrm{MHz}$ PC with $512 \mathrm{MB}$ of RAM was $128 \times 128$ pixels. The lower resolution was due to computer memory limitations. In order to compensate for this handicap, the original images were sampled. This sampled image was used for the analysis. a)

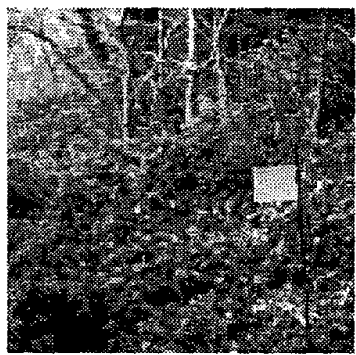

b)

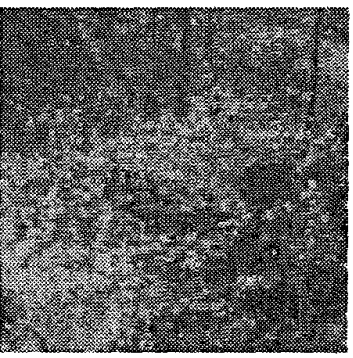

Fig. 2 - Results from applying the proposed method on the hyperspectral image shown in Fig. 1. a) One dimensional monochromatic output and b) three dimensional tri-chromatic output.

For comparison, both the original and modified hyperspectral images were also analyzed using principle component analysis (PCA). It has been shown [3] that PCA often associates important features in lower order eigenvalues. We compared the results of PCA and LLE and found strengths and weakness of each.

\section{RESULTS}

Fig. 1 shows an example of one of the hyperspectral images. The RGB image was extracted by multiplying all 31 channels together with each retinal spectral absorption curve. The image is of a small wooded area. The background consists of mostly trees, shrubs and various other wild plants. In the foreground is the white flag that was used for image calibration. It is supported by a short post.

Fig. 2 shows the result of applying the proposed method on the hyperspectral image from Fig. 1. In the a) the one dimensional output, different shades of gray correspond to not only variation in intensity, but also local variation in pixel spectra. In b) the three dimensional output, the colors correspond to different spectra. For example, the white flag appears as a relatively uniform color. In addition, the vegetation appears as predominantly one color and the tree bark appears as yet another color.

In Fig. 3, the hyperspectral image was decomposed using principle component analysis (PCA) for comparison. The first image appears to contain most of the image detail, which makes sense since the first score should display the most variation. Most of the detail lies in the background, where the shrubs and vegetation seem to stand out. The second and third basis images contain some features, but with much less detail than the first basis image. The second basis image shows twigs, bark and other tree debris, mostly in the lower right corner. In the third basis image, the 


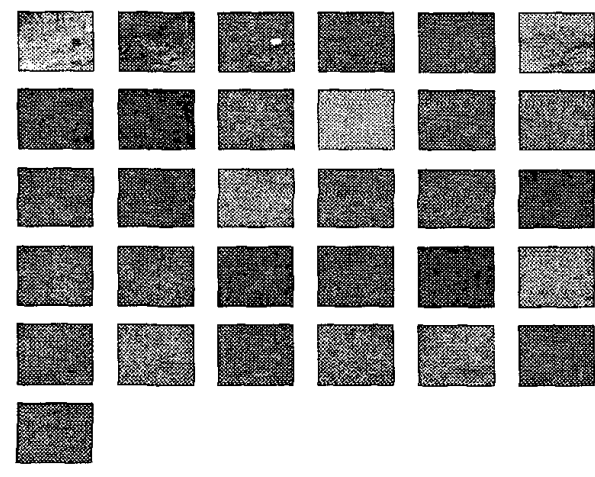

Fig.3 - Basis images derived from the original hyperspectral image using principle component analysis.

background is almost completely flat, yet the flag stands out prominently. Images beyond the third basis image are fairly flat and contain few if any recognizable features. Almost al of the variation in the hyperspectral image can be accounted for in the first three factors.

Fig. 4 shows a modified version of the hyperspectral image where a spectral irregularity was added to a region of the image near the top-left corner. Both a) the individual color channels of the hyperspectral image as well as b) a grayscale representation of the hyperspectral image, where all color channels were simply averaged together. In the third channel, the intensity was doubled and in the fourth channel, the intensity was set to zero. All other channels were left unchanged. Due to the nature of the spectral anomaly, the modification is virtually undetectable in the grayscale image. When the color channels are averaged, the spectral changes cancel out and the irregularity in the image becomes unnoticeable.

Fig. 5 shows the output of the k-means/LLE algorithm using the modified hyperspectral image. The distortion is clearly visible in both a) monochromatic and b) tri-chromatic output. In the monochromatic image, the anomaly appears as a lightened square. In the tri-chromatic output, the square is bright purple in the midst of a green and purple background. Still visible are all the same features visible in Fig. 2.

The modified hyperspectral image was analyzed using PCA and the resulting basis images are shown in Fig. 6. The results are similar to the results from the previous PCA, except the spectral anomaly is noticeable in the fifth and sixth basis images. In order to capture the features of the spectral anomaly, at least five basis images must be analyzed. In contrast, LLE was capable of showing the

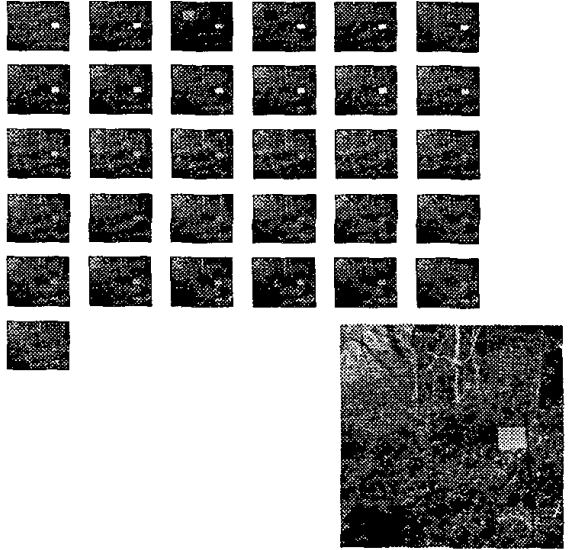

Fig.4 - Modified hyperspectral image. a) All color channels are displayed. Irregularities were added to the third and fourth channels. b) The hyperspectral image averaged to a single grayscale image.

anomaly in only one channel. The ability to discriminate among spectra only improved when using three channels.

\section{CONCLUSION}

The method successfully generated images where differences in the original spectra were reflected in differences in the output vector assignments. The kmeans/LLE algorithm efficiently grouped similar materials in the scene.

An additional benefit of mapping to a lower dimensional space was reduced data size. The images processed using LLE were several times small than the output images from PCA. Although PCA carries most of the important features in the first few basis images, there is no guarantee in which basis image a hidden feature will appear. The LLE algorithm generates images of known dimensionality, so the task of analyzing the output is more straightforward.

When spectral irregularities were added to a patch of the hyperspectral images, the method successfully generated color assignments that reflected differences in the spectra. The PCA was also capable of distinguishing the anomaly, but required searching higher basis images.

Although there are several benefits of using the LLE algorithm for this application, there are several disadvantages as well. First, several free parameters must be set, meaning that prior knowledge must be known about a scene. Parameters such as number of $\mathrm{k}$-means clusters and number of LLE neighbors require information about image composition. Another parameter, output dimensionality, also requires knowledge of image composition. In order to effectively map the spectra to lower dimensional vectors, one must know the dimensionality of the data. Mapping to too 
a)

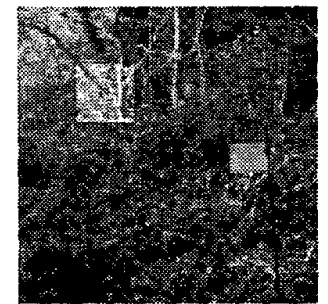

b)

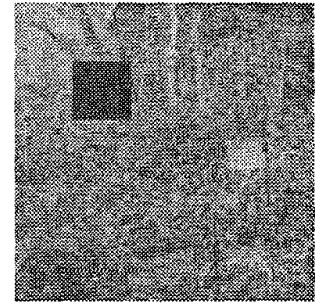

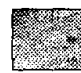
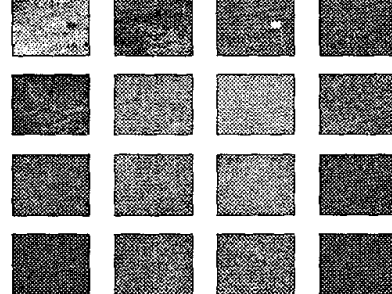

\section{西}
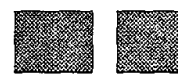

(1)

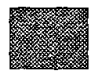

(1)

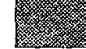

1.
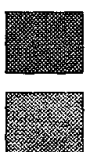
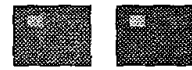

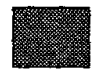
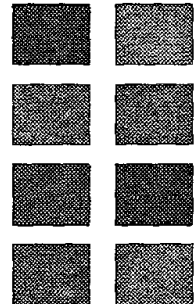

Fig.5 - The results from applying the proposed method on the modified hyperspectral image shown in Fig. 4. a) One dimensional monochromatic output and b) three dimensional tri-chromatic output.

many dimensions is wasteful, but mapping to too few dimensions will limit the output vector space.

A second problem is that of computing power. Although the images can be processed to an acceptable resolution, the memory requirements to solve the LLE weight functions are quite large. Although computing power increases over time, it is currently a limiting factor for processing higher resolution images.

A third consideration is the requirement for the LLE algorithm that the input be a well sampled manifold. Although images in a scene are likely to have spectra that vary from one spectrum to the next over a gradient, this cannot be guaranteed. If the image is composed of many disjoint objects and the resolution is low, the LLE algorithm may have difficulty mapping the spectra to proper outputs.

Despite these shortcomings, LLE offers promise for processing hyperspectral images. LLE represents a principled method for reducing the dimensionality of multidimensional images, while preserving the spatiospectral relationships among the pixels.

\section{ACKNOWLEDGMENT}

We would like to thank Lawrence Saul for helpful advice and guidance.

\section{REFERENCES}

[1] C.A. Parraga, G.J. Brelstaff, T. Troscianko and I. Moorhead, "Color and luminance information in natural scenes," JOSA A, Vol.15, p.563-569, March 1998.

[2] S.T. Roweis, L.K. Saul, "Nonlinear dimensionality reduction by locally linear embedding," Science, vol. 290, pp 2323-2326, December 2000.
Fig.6 - Basis images derived from the original hyperspectral image using principle component analysis.

[3] Schweizer, S.M., Moura, J.M.F., "Efficient detection in hyperspectral imagery," IEEE T an Image Processing, Vol. 10, pp $584-597$, April 2001.

[4] P. Bierwirth, R. Blewett, D. Huston, "Finding new mineral prospects with HYMAP; early results from a hyperspectral remote-sensing case study in west Pilbara," ASGO Research Newsletter, Vol 31, pp 29-31, November 1999.

[5] J.B. Tenenbaum, V.de Silva, J.C. Langford, " A Global Geometric Framework for Nonlinear Dimensionality Reduction," Science, vol 290, pp 2319-2323, December 2000.

[6] K. Staenz, J.-C Deguise, J. Chen, H. McNaim, T. Szeredi, M. McGovern, "The use of hyperspectral data for precision farming," ISPRS Comission VII Symposium, Budapest, Hungary, September 1-4, 1998

[7] H. McNaim, J.-C. Deguise, A. Pacheco, J. Shang, N. Rabe, "Estimation of crop cover and chlorophyll from hyperspectral remote sensing," Proceedings of the $23^{\text {Td }}$ Canadian Symposium on Remote Sensing, Ste. Foy, Quebec, August 20-24, 2001. 